\title{
Photoacoustic Spectroscopy
}

National Cancer Institute

\section{Source}

National Cancer Institute. Photoacoustic Spectroscopy. NCI Thesaurus. Code C103229.

The technique of measuring the effect of absorbed electromagnetic energy on solids, liquids and gases through detection of the acoustic waves released from the absorbing material. 